\title{
Collective Behaviour in a Square Lattice of Driven Duffing Resonators Coupled to van der Pol Oscillators
}

\author{
Michel F. Randrianandrasana \\ Nonlinearity and Complexity \\ Research Group \\ Aston University \\ Birmingham, UK, B4 7ET \\ Email: randrimf@aston.ac.uk
}

\author{
Xueyong Wei \\ School of Mechanical Engineering \\ University of Birmingham \\ Birmingham, UK, B15 2TT \\ Email: x.wei.2@bham.ac.uk
}

\author{
David Lowe \\ Nonlinearity and Complexity \\ Research Group \\ Aston University \\ Birmingham, UK, B4 7ET \\ Email: d.lowe@aston.ac.uk
}

\begin{abstract}
The global and local synchronisation of a square lattice composed of alternating Duffing resonators and van der Pol oscillators coupled through displacement is studied. The lattice acts as a sensing device in which the input signal is characterised by an external driving force that is injected into the system through a subset of the Duffing resonators. The parameters of the system are taken from MEMS devices. The effects of the system parameters, the lattice architecture and size are discussed.
\end{abstract}

\section{Introduction}

Real biosensor networks, such as the mammalian olfactory bulb as an exemplar system, and neurally-integrated autonomous communication networks in many nervous systems, share common characteristics and behaviours which are still not understood. For example (1), biophysical models to explain the gamma band oscillation in the olfactory bulb based on coupled subthreshold oscillators have been shown to exhibit synchronisation and coherence effects when driven with correlated inputs. Similarly, in recent models of temporal coding (2), competition for coherence driven by increased phase velocity of neural units in cases of coherent input from the connected neurons leads to different perspectives on assembly formation in collections of coupled oscillator neurons. The ability of a network of coupled nonlinear oscillators to develop emergent behaviour including topological organisation, associative memory and gamma band synchronisation, has already been used to illustrate a computational capability for abstract pattern recognition (3).

However, a major query is whether we can learn from such biological sensor and communication networks to design more robust biomorphically engineered sensor, communication and computing arrays in recent technologies such as micro and nano-mechanical silicon-based systems.

Motivated by the examples presented above, we are investigating the effects of Si-based sensor arrays of coupled microelectromechanical oscillators and resonators.

As already noted, in biological systems of coupled oscillatory devices, the interaction permits macroscopic temporal characteristics such as common locking of the phase or amplitude into synchronised states. In order to understand and predict the emergent phenomena of these systems, different mathematical models of chains and arrays of coupled resonators or oscillators have been constructed and studied (4). In a strongly coupled system, the synchronisation effects may be observed globally in all elements $(5 ; 6 ; 7)$ or locally in clusters of neighbouring elements $(8 ; 9 ; 10 ; 11)$ (cluster synchronisation).

Recent progress in micro/nano technology now enables scientists to design and manufacture coupled mechanical resonators to experimentally investigate the collective behaviour of such systems. For example, intrinsic localized modes were observed in a micromechanical oscillator array of about 220 coupled cantilevers (12). The formation and propagation range of the acoustic waves over two-dimensional array of $400 \mathrm{cou}-$ pled nano mechanical resonators were studied as a potential application in RF signal processing (13). It could be also expected that the collective behaviours of coupled oscillators can be utilized in novel sensing devices (14), optomechanical signal processing devices (15) or artificial intelligence decision-making (16).

To extend these recent activites and also to link to recent mathematical work on coupled oscillator array systems, in this paper, the collective behaviour of alternating Duffing resonators and van der Pol oscillators elastically coupled in a square lattice is numerically studied with typical MEMS parameters. The architecture of the lattice of resonators and oscillators along with some characterisations of synchronisation will be described first. Then the effect of parameter variation on the behaviour of the system will be discussed followed by different configurations and sizes of the lattice.

\section{Experimental setup}

The architecture of the network of oscillators consists of a square lattice of alternating Duffing resonators and van der Pol oscillators in a checkerboard configuration as shown in 
Figure 1. The dimensionless equations governing the coupled systems are:

$$
\begin{aligned}
\ddot{x}+\gamma \dot{x}+x+\delta x^{3}= & \kappa\left(x_{i, j+1}+x_{i, j-1}+x_{i-1, j}\right. \\
& \left.+x_{i+1, j}-4 x_{i, j}\right) \\
\ddot{x}+\gamma\left(x^{2}-1\right) \dot{x}+x= & \kappa\left(x_{i, j+1}+x_{i, j-1}+x_{i-1, j}\right. \\
& \left.+x_{i+1, j}-4 x_{i, j}\right),
\end{aligned}
$$

where $\gamma$ is the damping of the Duffing resonator and the van der Pol oscillator, $\delta$ is the cubic nonlinear spring constant of the Duffing resonator and $\kappa$ is the coupling strength between the resonators and oscillators. The element $x_{i, j}$ represents the displacement of the resonator or oscillator located at the $i$-th row and $j$-th column in the square lattice.

Since the array of oscillators will act as a sensing device, it is usually subject to some external signal that can be represented as a driving force or energy for the array. The equations for the driven Duffing resonator and van der Pol oscillator respectively are therefore given below:

$$
\ddot{x}+\gamma \dot{x}+x+\delta x^{3}=F_{d} \cos (\Omega \tau),
$$

and

$$
\ddot{x}+\gamma\left(x^{2}-1\right) \dot{x}+x=F_{d} \cos (\Omega \tau),
$$

where $F_{d}$ is the normalised driving force.

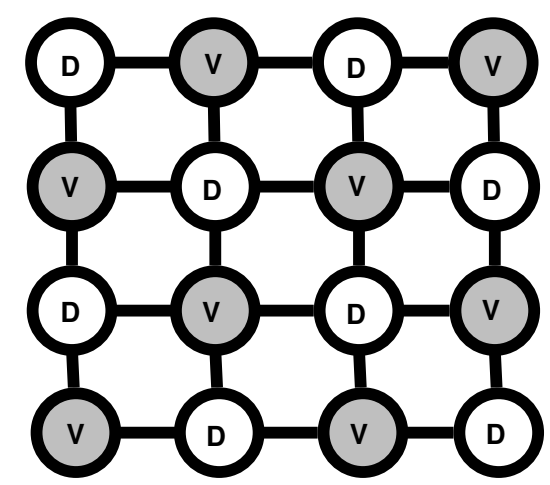

Fig. 1. Square lattice of alternating Duffing resonators and van der Pol oscillators.

In this paper, the synchronous behaviour of the resonators and oscillators in the square lattice will be characterised in terms of their global and local synchronisations. In the first case, the resonators and oscillators will mainly be considered as one group whereas the elements in the array can be gathered in different subgroups in the second case. The global coherence of the array will be measured by a synchronisation index based on the normalised entropy (17):

$$
\tilde{\rho}=\frac{S_{\max }-S}{S_{\max }},
$$

where $S=-\sum_{k=1}^{N} \rho_{k} \ln \rho_{k}$ is the entropy of the distribution of the instantaneous phase or the amplitude of the oscillators, which is given by their respective histograms from which the probabilities $\rho_{k}$ are computed, and $S_{\max }=\ln N$, where $N$ is the number of bins of the histogram. $0 \leq \tilde{\rho} \leq 1$ and $\tilde{\rho}=0$ means that the array is not coherent or in total disorder, whereas $\tilde{\rho}=1$ indicates that all the oscillators in the array are perfectly synchronised. Besides full synchronisation it is also interesting to consider another aspect of synchronisation namely the cluster synchronisation where the oscillators involved in the same cluster have identical temporal dynamics and completely synchronise between each other. Unlike the global synchronisation case, no cluster synchronisation index is available yet. Therefore, only a visual inspection of the displacements and phases of the elements in the lattice will be carried out. To this end, phase images and polar plots of the elements of the square lattice will be produced. The phase and polar plots will be complementary as the phase images will help to locate the resonators and oscillators within the array while the polar plots will display both the amplitudes and phases of the elements. In the following, the color scale in the phase images lies between $-\pi$ and $\pi$ and the polar plots are normalised in amplitude which means that the maximum amplitude corresponds to a distance of 1 in the unit circle.

\section{Parameter effects}

We investigate the effect of the normalised coupling strength $\kappa$ between the oscillators, the amplitude $F_{d}$ of the driving force that is injected from one edge of the lattice and the quality factor $Q$ of each element of the lattice. The quality factor $Q$ of the oscillators and resonators is directly related to their damping $\gamma$ through $\gamma=1 / Q$. It is also important to stress that only the Duffing resonators are driven since they absorb energy whereas the van der Pol oscillators are self-sustained.

The parameters are given in their dimensionless values. In the context of MEMS devices, the coupling strength $\kappa$ ranges from 0.001 to 1000 . The amplitude $F_{d}$ of the driving force is assumend to be in the range 0.3 to 30 . The quality factor $Q$ lies within the interval $[10,1000]$, where the lowest and biggest $Q$ values corresponds to the devices being in air and in vacuum respectively.

The coupling strength between the elements of the array has a counter-intuitive effect on the global coherence of the amplitudes of the resonators and oscillators. The lower the coupling the more coherent is the system as shown in Figure 2 . The value of the coupling strengthhas no clear relaton with the global phase coherence of the elements although the global phase coherence of the system tends to converge quicker with higher coupling values. More detailed information regarding the cluster synchronisation of the elements in the lattice are given by the phase and polar plots in Figure 3. It can clearly be seen that with low coupling $(\kappa=1)$ the behaviour of the elements is more like random. However, with high coupling $(\kappa=100)$ the phases of the resonators and oscillators located on odd rows, and respectively on even rows, are synchronised between them. These two distinct clusters are shown in both the phase and amplitude spaces by the polar plot of Figure $3(d)$. When the coupling strength is extremely high $(\kappa=1000)$ 


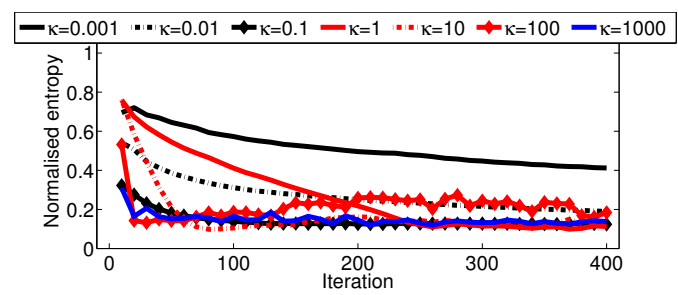

(a) Phase

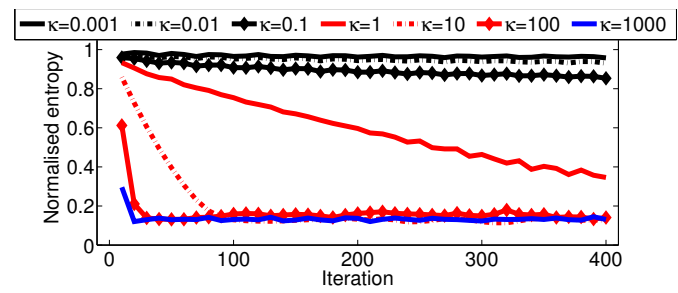

(b) Amplitude

Fig. 2. Coupling strength effect on the global coherence of the square lattice. The phase coherence does not seem to have a direct relation with the coupling strength although for a very high coupling $(\kappa=10000)$ the phases of the elements of the lattice tend to converge faster. On the other hand, the amplitude coherence clearly depends on the coupling strength value: the higher the coupling strength, the slower the convergence and the less synchronised are the elements of the lattice.

the phases and amplitudes of the elements of the lattice are almost homogeneous (plots not shown) as can be expected.

The amplitude of the driving force has an opposite effect on the global coherence of the phase and amplitude of the system. More precisely, the higher the amplitude of the driving force the less coherent is the phase of the system whereas the opposite effect is observed for the amplitude of the elements of the lattice. However it should be noted that the difference in global phase coherence is not very significant as the amplitude of the driving force is multiplied by ten. By looking at the cluster synchronisation aspect, it can be observed that increasing the amplitude of the driving force will result in a randomisation of the phases of the resonators and oscillators as depicted by Figure 4. The latter result is also valid for the amplitudes of the elements although the plots are not shown.

The global phase coherence of the lattice is not sensitive to the value of the quality factor. However, for low quality factor value $(Q=10)$ the global amplitude coherence of the system displays better synchronisation behaviour than higher values of $Q$ as shown by Figure 5. In contrast to the previous full synchronisation results, more distinguishable amplitude and phase clusters appear in the lattice when the quality factor value is high $(Q=1000)$ as can be seen in Figure 6. It should be noted however that for small $Q$ value, the Duffing resonators and van der Pol oscillators have two distinct behaviours. The system is not sensitive to initial conditions (plots not shown).

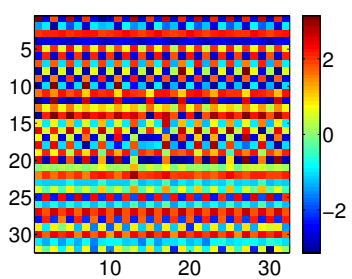

(a) $\kappa=1$

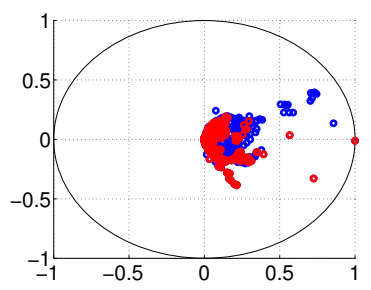

(c) $\kappa=1$

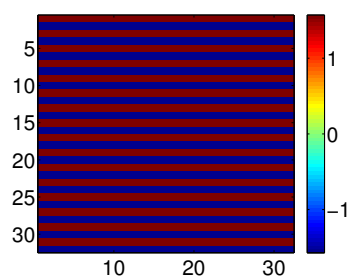

(b) $\kappa=100$

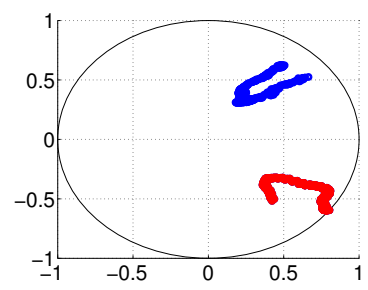

(d) $\kappa=100$
Fig. 3. Phase and polar plots of a square lattice of 32 by $32 \mathrm{x}$-coupled alternating Duffing resonators and van der Pol oscillators driven from the top edge with an energy whose amplitude is $F_{d}=0.3$. The figures are plotted at $t=3600$ with different coupling strength values. At low coupling $(\kappa=1)$ both the phases and amplitudes of the resonators and oscillators seem to be random. For high coupling $(\kappa=100)$ the resonators and oscillators on odd rows are synchronised between them and so are the resonators and oscillators located on even rows. When the coupling is extremely high $(\kappa=1000)$ the Duffing resonators and the van der Pol oscillators are both synchronised in phase and amplitude (plots not shown).

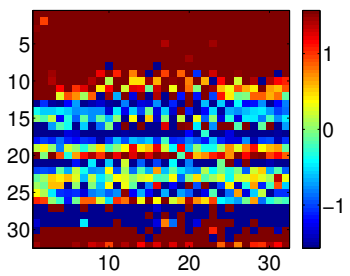

(a) $F_{d}=3$

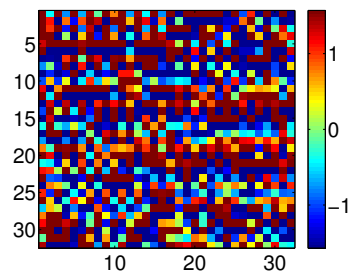

(b) $F_{d}=30$
Fig. 4. Phase plots of a square lattice of 32 by $32 x-$ coupled alternating Duffing resonators and van der Pol oscillators at a coupling strength of $\kappa=10$. The array is driven from the top edge of the lattice and the figures are plotted at $t=3600$ with different amplitudes of the driving force. It can be noticed here that when the lattice is driven ten times higher, then the phases of the elements tend to desynchronise. 


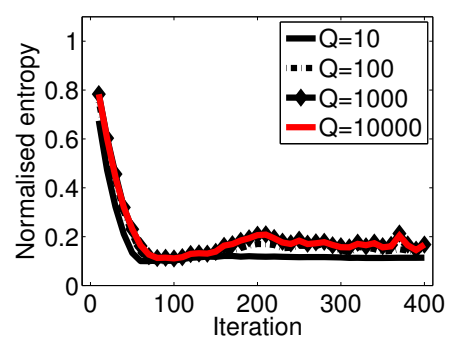

(a) Phase

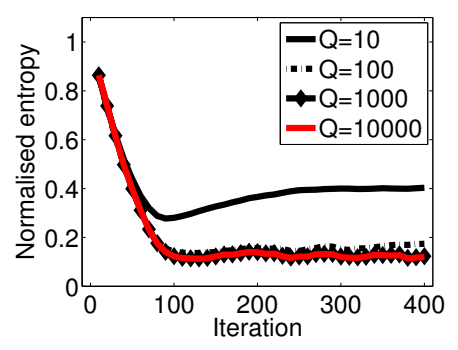

(b) Amplitude

Fig. 5. Relation between the quality factor $Q$ of the elements of the square lattice and its global coherence. It can be noticed that the lower the value of $Q$ the worse the phase coherence. A possible explanation of this observation is that for low $Q$ values, the separation between the behaviour of the Duffing resonators and van der Pol oscillators is clearly seen as depicted by Figure $6(c)$. In contrast, the amplitude coherence of the lattice is improved as the value of $Q$ is decreased.

\section{Architecture and size effects}

In the previous section, a square lattice of alternating Duffing resonators and van der Pol oscillators has been studied. In this section, we investigate the behaviour of the coupled systems when we permute the Duffing resonators and the van der Pol oscillators in the lattice.

The two different architectures, characterised by a lattice of alternating Duffing resonators and van der Pol oscillator on one hand and alternating van der Pol oscillators and Duffing resonators on the other hand, have almost the same behaviour in terms of the global coherence of their phases and amplitudes (plots not shown). However they have different cluster synchronisation properties as illustrated in Figure 7. In the case of a lattice composed of alternating Duffing resonators and van der Pol oscillators, different subgroups can be observed in which the phases of the Duffing resonators and van der Pol oscillators are synchronised. In the other case, the Duffing resonators and van der Pol oscillators behace as two separate subgroups.

It is also interesting to be able to understand the change of behaviour as the size of the lattice varies. In particular, the effect of having an odd number of oscillators and resonators is considered as opposed to the previous case where the

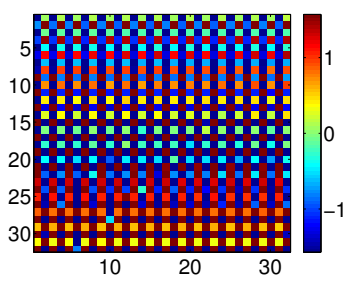

(a) $Q=10$

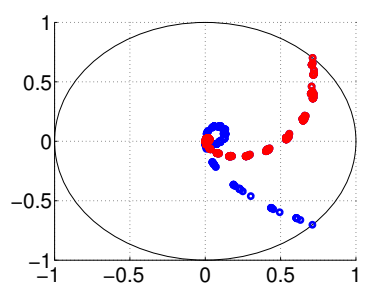

(c) $Q=10$

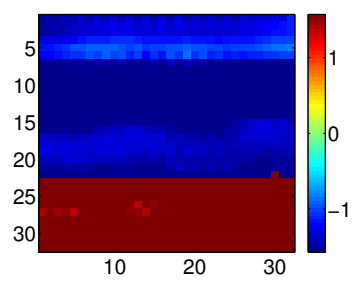

(b) $Q=1000$

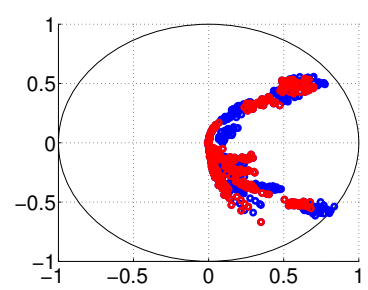

(d) $Q=1000$
Fig. 6. Phase and polar plots of a square lattice of 32 by $32 \mathrm{x}$-coupled alternating Duffing resonators and van der Pol oscillators driven from the top edge with an energy whose amplitude is $F_{d}=0.3$. The coupling between the elements is $\kappa=10$ and the figures are plotted at $t=3600$ with different values of the quality factor $Q$. The figures show that when $Q$ is small $(Q=10)$ the Duffing resonators (red circles) and the van der Pol oscilators (blue circles) have two distinct behaviours. However at a high quality factor $(Q=1000)$ they tend to synchronise between them.

dimension of the square lattice is even. From the plots in Figure 8 , it can be concluded that the global phase and amplitude coherence of the lattice decrease as the size of the lattice is increased when the number of elements in the lattice is even. A different behaviour can be noticed for odd number of elements. In the cluster synchronisation case, it can be noticed that the bigger the lattice the more clusters are present for even number of elements (Figure 9). In contrast, when the number of elements is odd, no identifiable clusters can be observed but the elements have disparate phases and almost random amplitudes.

\section{Conclusion}

The global and local synchronisations of a square lattice of alternating Duffing resonators and van der Pol oscillators have been studied numerically. Such a lattice can serve as a sensing device where the input signal is an external driving force injected on the Duffing resonators. The parameters of the resonators and oscillators derive from real micro-electromechanical system (MEMS) devices. It has been found, unsurprisingly, that the higher the coupling strength value between the elements of the lattice the more synchronised they will become. In contrast, when the amplitude of the driving force is increased the elements of the lattice tend to randomise. 


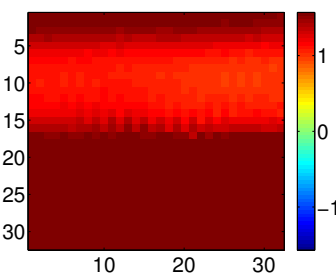

(a) Duff-VdP

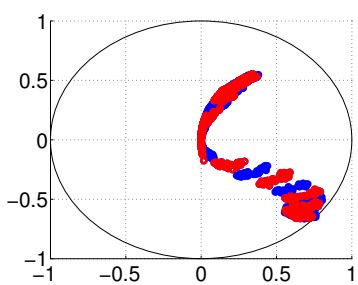

(c) Duff-VdP

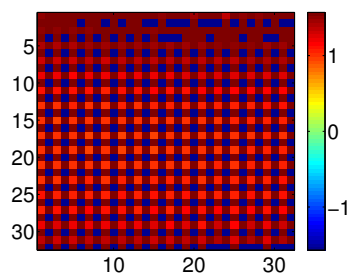

(b) VdP-Duff

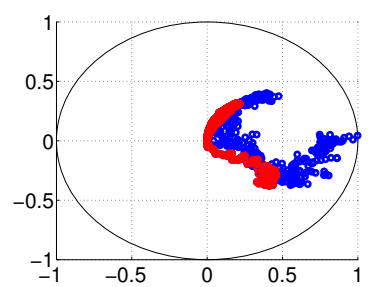

(d) VdP-Duff
Fig. 7. Phase and polar plots of a square lattice of 32 by $32 \mathrm{x}$-coupled alternating Duffing resonators and van der Pol oscillators, and alternating van der Pol oscillators and Duffing resonators at a coupling strength of $\kappa=40$. The lattice is driven from the top edge with an energy whose amplitude is $F_{d}=0.3$. The figures are plotted at $t=3600$. The phases of the elements are more synchronised in the first case (Duff-VdP) and it can observed from the corresponding polar plot that some the Duffing resonators and van der Pol oscillators evolve in disctint subgroups. In the second case (VdP-Duff), the Duffing resonators tend to group between them and so do the van der Pol oscillators.

With high quality factor values the synchronisation between the elements is favoured whereas a low quality factor value will result in two distinct behaviours of the resonators and oscillators.

Architecture and finite size effects are apparent. A permutation of the resonators and oscillators results in a lattice with different properties. The behaviours of the elements in these two types of lattices are different in that the resonators and oscillators can be synchronised in one case while they evolve independently in the other case. Different emergent clusters of synchronisation can be observed with different array sizes composed of an even number of elements. However, no distinguishable clusters of synchronisation appear when the dimension of the lattice is odd. This suggests that the unit composed of a Duffing resonator coupled with a van der Pol oscillator represents an important entity.

We have observed different behaviours depending upon how it is driven. Driving from the top edge, the resonators and oscillators tend to synchronise by rows provided that the coupling strength is high enough. In the second case, driving from a corner device, a symmetric behaviour of the elements of the lattice can be seen where the axis of symmetry is given by the leading diagonal. Different initial conditions have also

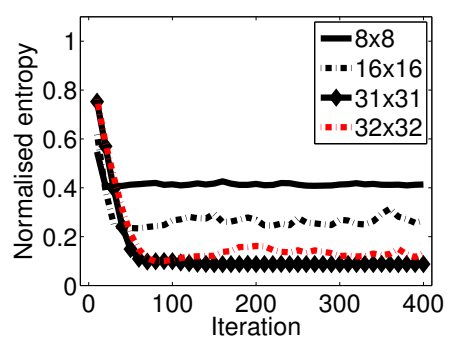

(a) Phase

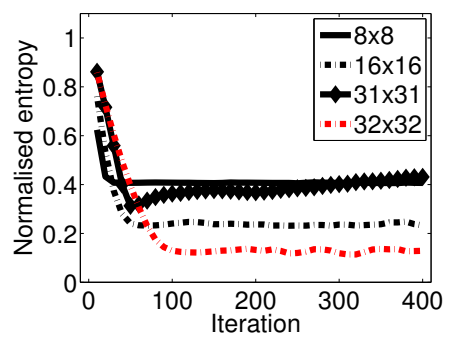

(b) Amplitude

Fig. 8. Lattice size effect on the global coherence of the elements. In the case of an even number of elements, the smaller the lattice, the quicker the convergence and the better the global coherence of the phase and amplitude of the elements in the lattice. The global synchronisation of the amplitudes of the elements is however better when the number of elements is odd (here $31 \times 31$ ) compared to the even number elements $(32 \times 32)$.

been used but the system is not sensitive to initial conditions.

We have also commented and illustrated that both global and cluster synchronisations are both possible in the same heterogeneous architecture.

All of these observations are useful in supporting our original aim from the Introduction: to engineer more robust biomorphically-motivated sensor, communication and computing arrays in MEMS/NEMS technologies. We know that coupled oscillator arrays in biology exhibit a range of behaviours we have yet to exploit. This study shows we can expect similar patterns of emergence in real MEMS arrays. We are currently constructing chains and small arrays of such devices.

\section{Acknowledgement}

The authors acknowledge the financial support of this work from the Engineering and Physical Sciences Research Council (EPSRC) under the project number EP/D00036X/1 and EP/D501032/1. 


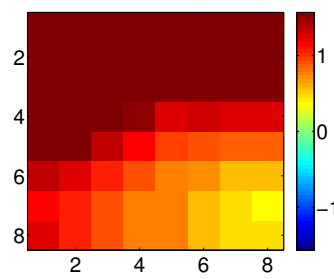

(a) $8 \times 8$

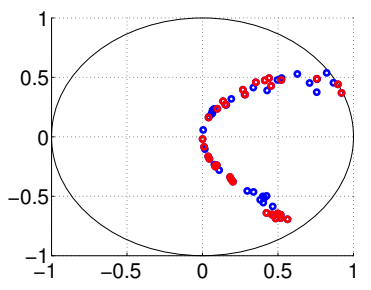

(c) $8 \times 8$

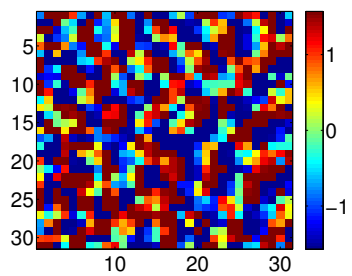

(b) $31 \times 31$

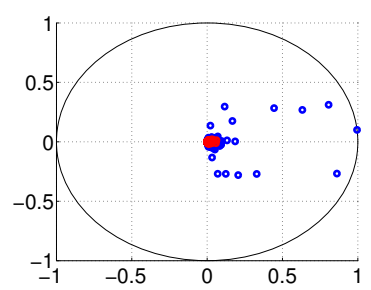

(d) $31 \times 31$
Fig. 9. Phase and polar plots of a square lattice of 32 by $32 \mathrm{x}$-coupled alternating Duffing resonators and van der Pol oscillators at a coupling strength of $\kappa=10$. The array is driven from the top edge of the lattice and the figures are plotted at $t=3600$ for different sizes of the lattice. It can be observed that for small $(8 \times 8)$ and medium (16x16) array (plots not shown) sizes, the phases and amplitudes of the Duffing resonators and the van der Pol oscillators are in synchrony. In particular, the smaller the lattice, the better is the synchronisation. However, when the dimension on the lattice is odd, 31 by 31 in this case, the synchronisation is lost and it can also be noticed that the amplitudes of the Duffing resonators are quite small compared to those of the van der Pol oscillators.

\section{References}

[1] J. N. Breaa, L. M. Kayb, and N. J. Kopella, "Biophysical model for gamma rhythms in the olfactory bulb via subthreshold oscillations," PNAS, vol. 106, no. 51, pp. 21 954-21 959, December 2009.

[2] T. Burwick, "Temporal coding: competition for coherence and new perspectives on assembly formation," in IJCNN'09: Proceedings of the 2009 international joint conference on Neural Networks. Piscataway, NJ, USA: IEEE Press, 2009, pp. 3467-3476.

[3] M. Ursino, E. Magosso, and C. Cuppini, "Recognition of abstract objects via neural oscillators: interaction among topological organization, associative memory and gamma band synchronization," Trans. Neur. Netw., vol. 20, no. 2, pp. 316-335, 2009.

[4] J. A. Acebron, L. L. Bonilla, C. J. Pérez Vicente, F. Ritort, and R. Spigler, "The kuramoto model: A simple paradigm for synchronization phenomena," Rev. Mod. Phys., vol. 77, no. 1, pp. 137-185, Apr 2005.

[5] W. Wang and J.-J. E. Slotine, "On partial contraction analysis for coupled nonlinear oscillators," Biological
Cybernetics, vol. 92, no. 1, pp. 38-53, 2004.

[6] C.-H. Chiu, W.-W. Lin, and C.-S. Wang, "Synchronization in a lattice of coupled oscillators with various boundary conditions," Nonlinear Analysis, vol. 46, pp. 213-229, 2001.

[7] V. S. Afraimovich, S. N. Chow, and J. K. Hale, "Synchronisation in lattices of coupled oscillators," Physica D, vol. 103, pp. 445-1490, 1997.

[8] K. Kaneko, "Clustering, coding, switching, hierarchical ordering, and control in a network of chaotic elements," Physica D, vol. 41, pp. 137-172, 1990.

[9] F. Xie and G. Hu, "Spatiotemporal periodic and chaotic patterns in a two-dimensional coupled map lattice system," Physical Review E, vol. 55, pp. 79-86, 1997.

[10] V. N. Belykh, I. V. Belykh, and M. Hasler, "Hierarchy and stability of partially synchronous oscillations of diffusively coupled dynamical systems," Physical Review $E$, vol. 62, no. 5, pp. 6332-6345, 2000.

[11] V. N. Belykh and E. Mosekilde, "Cluster synchronization modes in an ensemble of coupled chaotic oscillators," Physical Review E, vol. 63, p. 036216, 2001.

[12] M. Sato, B. E. Hubbard, A. J. Sievers, B. Ilic, D. A. Czaplewski, and H. G. Craighead, "Observation of locked intrinsic localized vibrational modes in a micromechanical oscillator array," Phys. Rev. Lett., vol. 90, no. 4, p. 044102, Jan 2003.

[13] M. K. Zalalutdinov, J. W. Baldwin, M. H. Marcus, R. B. Reichenbach, J. M. Parpia, and B. H. Houston, "Two-dimensional array of coupled nanomechanical resonators," Appl. Phys. Lett., vol. 88, p. 1143504, 2006.

[14] P. A. Truitt, J. B. Hertzberg, C. C. Huang, and K. C. S. Kamil L. Ekinci and, "Efficient and sensitive capacitive readout of nanomechanical resonator arrays," Nano Letters, vol. 7, no. 1, pp. 120-126, 2007.

[15] E. Buks and M. L. Roukes, "Electrically tunable collective response in a coupled micromechanical array," Journal of MicroElectroMechanical Systems, vol. 11, no. 6, pp. 802-807, 2002.

[16] R. Rand and J. Wong, "Dynamics of four coupled phaseonly oscillators," Communications in Nonlinear Science and Numerical Simulation, vol. 13, no. 3, pp. 501-507, 2008.

[17] P. Tass, M. G. Rosenblum, J. Weule, J. Kurths, A. S. Pikovsky, J. Volkmann, A. Schnitzler, and H.-J. Freund, "Detection of n:m phase locking from noisy data: application to magnetoencephalography," Physical Review Letters, vol. 81, no. 15, pp. 3291-3294, 1998. 